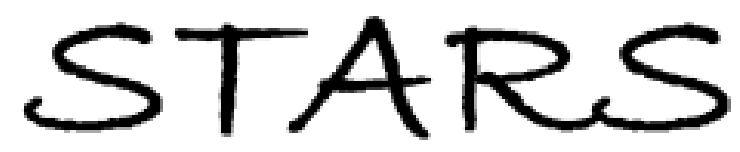

University of Central Florida

STARS

$1-1-2007$

\title{
Electrode architecture in tuning room temperature sensing kinetics of nanomicrointegrated hydrogen sensor
}

\author{
Sameer Deshpande \\ University of Central Florida \\ Sudipta Seal \\ University of Central Florida \\ Peng Zhang \\ University of Central Florida \\ Hyoung J. Cho \\ University of Central Florida \\ Nathan Posey \\ University of Central Florida \\ Find similar works at: https://stars.library.ucf.edu/facultybib2000 \\ University of Central Florida Libraries http://library.ucf.edu
}

This Article is brought to you for free and open access by the Faculty Bibliography at STARS. It has been accepted for inclusion in Faculty Bibliography 2000s by an authorized administrator of STARS. For more information, please contactSTARS@ucf.edu.

\section{Recommended Citation}

Deshpande, Sameer; Seal, Sudipta; Zhang, Peng; Cho, Hyoung J.; and Posey, Nathan, "Electrode architecture in tuning room temperature sensing kinetics of nanomicrointegrated hydrogen sensor" (2007). Faculty Bibliography 2000s. 7025.

https://stars.library.ucf.edu/facultybib2000/7025

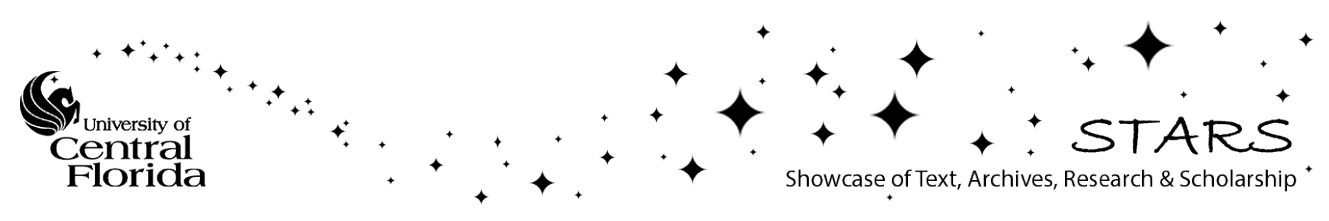




\section{Electrode architecture in tuning room temperature sensing kinetics of nanomicrointegrated hydrogen sensor}

Cite as: Appl. Phys. Lett. 90, 073118 (2007); https://doi.org/10.1063/1.2644276

Submitted: 04 December 2006 . Accepted: 16 January 2007 . Published Online: 16 February 2007

Sameer Deshpande, Sudipta Seal, Peng Zhang, Hyoung J. Cho, and Nathan Posey

ARTICLES YOU MAY BE INTERESTED IN

Gas sensing properties of defect-controlled ZnO-nanowire gas sensor

Applied Physics Letters 93, 263103 (2008); https://doi.org/10.1063/1.3046726

Magnetoresistance sensor with an out-of-plane magnetized sensing layer Applied Physics Letters 87, 022504 (2005); https://doi.org/10.1063/1.1957111

Sound-resonance hydrogen sensor

Applied Physics Letters 82, 4590 (2003); https://doi.org/10.1063/1.1586994

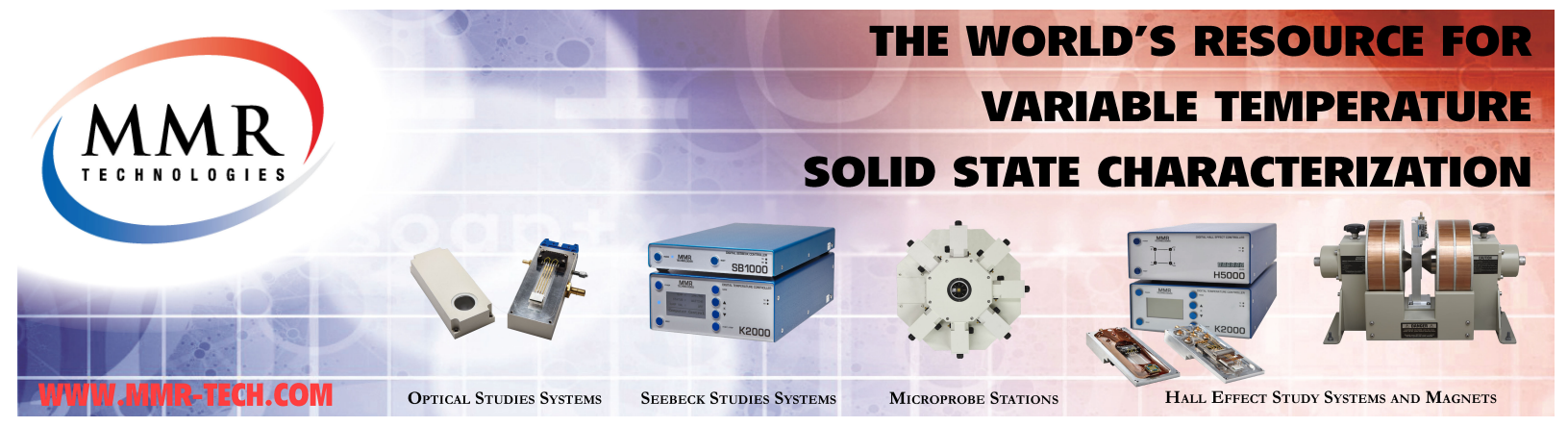




\title{
Electrode architecture in tuning room temperature sensing kinetics of nanomicrointegrated hydrogen sensor
}

\author{
Sameer Deshpande and Sudipta Seal ${ }^{a)}$ \\ Surface Engineering and Nanotechnology Facility (SNF), University of Central Florida, 4000 Central \\ Florida Blvd., Orlando, Florida 32816; Advanced Materials Processing and Analysis Center (AMPAC), \\ University of Central Florida, 4000 Central Florida Blvd., Orlando, Florida 32816; Nanoscience \\ and Technology Center, University of Central Florida, 4000 Central Florida Blvd., Orlando, Florida 32816; \\ and Mechanical, Material and, Aerospace Engineering (MMAE) Department, University of Central \\ Florida, 4000 Central Florida Blvd., Orlando, Florida 32816 \\ Peng Zhang and Hyoung J. Cho \\ Mechanical, Material and, Aerospace Engineering (MMAE) Department, University of Central Florida, \\ 4000 Central Florida Blvd., Orlando, Florida 32816 \\ Nathan Posey \\ Electrical and Computer Engineering, University of Central Florida, 4000 Central Florida Blvd., Orlando, \\ Florida 32816
}

(Received 4 December 2006; accepted 16 January 2007; published online 16 February 2007)

\begin{abstract}
A diffusion model is proposed for elucidating the effect of interelectrode distance modulation on conductance change of a nanomicrointegrated hydrogen sensor at room temperature. Both theoretical and experimental results showed a faster response upon exposure to hydrogen when sensor electrode gap was smaller. Also, a linear increase in the sensor sensitivity from 500 to 80000 was observed on increasing the electrode spacing from 2 to $20 \mu \mathrm{m}$. The improvement in sensitivity is attributed to the higher reactive sites available for the gaseous species to react on the sensor surface. (ㅇ 2007 American Institute of Physics. [DOI: 10.1063/1.2644276]
\end{abstract}

Semiconductor nano-metal-oxide (e.g., $\mathrm{SnO}_{2}$ ) based thin film sensors have been widely used for the detection of hazardous gases. ${ }^{1}$ Currently, there is a lot of interest in developing a room temperature hydrogen sensor for various practical applications. ${ }^{2}$ Development of a practical sensor device requires the optimization of the sensitivity, response, and recovery time. Film thickness, particle size, porosity, metal oxide dopants, and operating temperature are some of the key material characteristics for optimum sensor performance. ${ }^{3}$ The activation energy for the gas-nano-metal-oxide reaction increases with reducing sensor operating temperature. This increased the activation energy that may impede the overall sensor response at lower temperatures (especially at room temperature). In such a scenario, variation in the design parameters in sensor electrode architecture cannot be underestimated in obtaining enhanced sensor performance at lower temperatures. This letter presents the influence of electrode geometry on tuning the room temperature sensing kinetics of a doped nano-tin-oxide thin film as a hydrogen sensor.

A microelectromechanical system (MEMS) hydrogen sensor device with varying electrode spacing $(g=2-20 \mu \mathrm{m})$ and constant electrode length $(l=1000 \mu \mathrm{m})$ was fabricated ${ }^{4}$ [Fig. 1(a)]. Advantages of fabricating microinterdigitated electrodes include (i) precise control over the geometry and dimension allowing reproducible and low cost devices and (ii) large sensing area with small spacing between electrodes (improved sensitivity compared to macroelectrodes). The MEMS device was coated with a sol-gel derived thin film of indium doped nanostructured tin oxide ${ }^{4}$ and subsequently wire bonded to an integrated circuit chip.

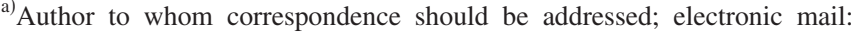
sseal@mail.ucf.edu
}

Figure 1(c) shows the cross section of the nano-tin-oxide coated gold electrode, engraved using a site-selective focused ion-beam (FIB) milling technique. The thickness of the tin oxide film is about $150 \mathrm{~nm}$ [Fig. 1(b)]. The sensor was tested in $900 \mathrm{ppm} \mathrm{H}_{2}$ at ambient conditions $\left(25^{\circ} \mathrm{C}\right)$.

When freshly prepared $\mathrm{SnO}_{2}$ thin film is exposed to air, oxygen ions $\left(\mathrm{O}^{-}\right.$or $\left.\mathrm{O}_{2}^{-}\right)$adsorb on the surface by capturing electrons from the conduction band of $\mathrm{SnO}_{2}$. This results in the formation of an electrical double layer (space charge layer) on the particle surface, which consists of negatively charged oxygen ions on the surface and positively charged metal ions on the inside. ${ }^{5,6}$ This increases the potential barrier for electron conduction within the film. When a reducing
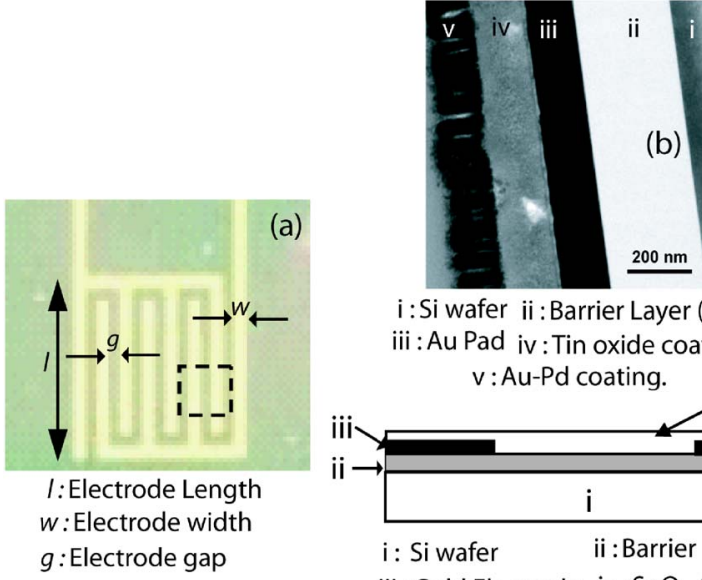

$\mathrm{i}:$ Si wafer ii : Barrier Layer $\left(\mathrm{SiO}_{2}\right)$

iii : Au Pad iv : Tin oxide coating $v:$ Au-Pd coating.

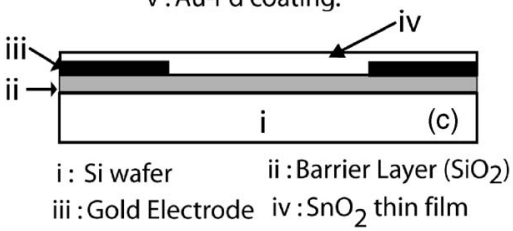

FIG. 1. (Color online) (a) Image showing the nano-tin-oxide coated microchip, (b) FIB cross section of the nano-tin-oxide coated microchip, and (c) side of the metal oxide coating and electrode arrangement. 
gas $\left(\mathrm{H}_{2}\right)$ comes in contact with a thin film, it is oxidized by reacting with $\mathrm{O}_{\text {ads }}^{-}$or $\mathrm{O}_{2 \text { ads }}^{-}$species, and electrons are reintroduced into the electron depletion layer, leading to decrease in the potential barrier and decrease in the film resistance. ${ }^{7}$

$$
\begin{aligned}
& \mathrm{H}_{2(\mathrm{~atm})}+\mathrm{O}_{(\mathrm{ads})}^{-} \rightarrow \mathrm{H}_{2} \mathrm{O}_{(\mathrm{ads})}+e^{-}, \\
& \mathrm{H}_{2_{(\mathrm{atm})}}+\frac{1}{2} \mathrm{O}_{2(\mathrm{ads})} \rightarrow \mathrm{H}_{2} \mathrm{O}_{(\mathrm{ads})}+e^{-} .
\end{aligned}
$$

Exposure of the thin film tin oxide lying above the coplanar electrodes [see Fig. 1(c)] to reducing gas results in the adsorption and simultaneous diffusion to the reactive sites. The diffusion of hydrogen inside the film can be considered as one dimensional gas diffusion in a medium bounded by two parallel planes [Fig. 1; thickness $\left(x_{0}\right)$ ]. The rate of reaction inside the film is effectively controlled by the diffusion rate of hydrogen. The ratio of the gas concentration inside the film at any point to the concentration of gas on the film surface can be defined as ${ }^{8}$

$$
C=\frac{c}{c_{s}} .
$$

$c$ is the concentration at a distance $x$ inside the film and the gas of initial surface concentration $\left(c_{s}\right)$ diffuses into the thin film of tin oxide (having thickness $x_{0}$ ), lying on the coplanar electrodes, with the following boundary conditions:

$$
C=c / c_{s}=1 \quad \text { when } x=x_{0} \text { (on the surface of the film) }
$$

and $C=c / c_{s}=0$ when $x=0$ (underneath the film).

The solution satisfying the above conditions becomes ${ }^{8}$

$$
C=\frac{c}{c_{s}}=1-\frac{4}{\pi} \sum_{n=0}^{\infty} \frac{(-1)^{n}}{2 n+1} e^{-D(2 n+1)^{2} \pi^{2} t / 4 x_{0}^{2}} \cos \frac{(2 n+1) \pi x}{2 x_{0}},
$$

where $D$ is the diffusion coefficient of the diffusing gas and $t$ is time.

Introducing the dimensionless parameters in Eq. (3) as follows: ${ }^{8}$

$$
T=\frac{D t}{x_{0}^{2}},
$$

Eq. (3) becomes

$C=\frac{c}{c_{s}}=1-\frac{4}{\pi_{n=0}} \sum_{n=1}^{\infty} \frac{(-1)^{n}}{2 n+1} e^{-(2 n+1)^{2} \pi^{2} T / 4} \cos \frac{(2 n+1) \pi X}{2}$.

An analytical solution for Eq. (4) is shown in Fig. 2(a), which depicts the variation in concentration at different exposure times at different film thickness. In the present case, the length of electrodes was kept constant $(1000 \mu \mathrm{m})$ for all sensors and the edge effect was neglected (since coating width $\gg$ coating thickness). According to Ohm's law the current is proportional to the applied electric field $(E)$. The current density $J$ (current per unit area) inside the nano-tinoxide film is a function of the conductivity $(\sigma)$ and can be defined as ${ }^{9}$

$$
J=\sigma E,
$$

while the current flowing through the device is given by ${ }^{10}$
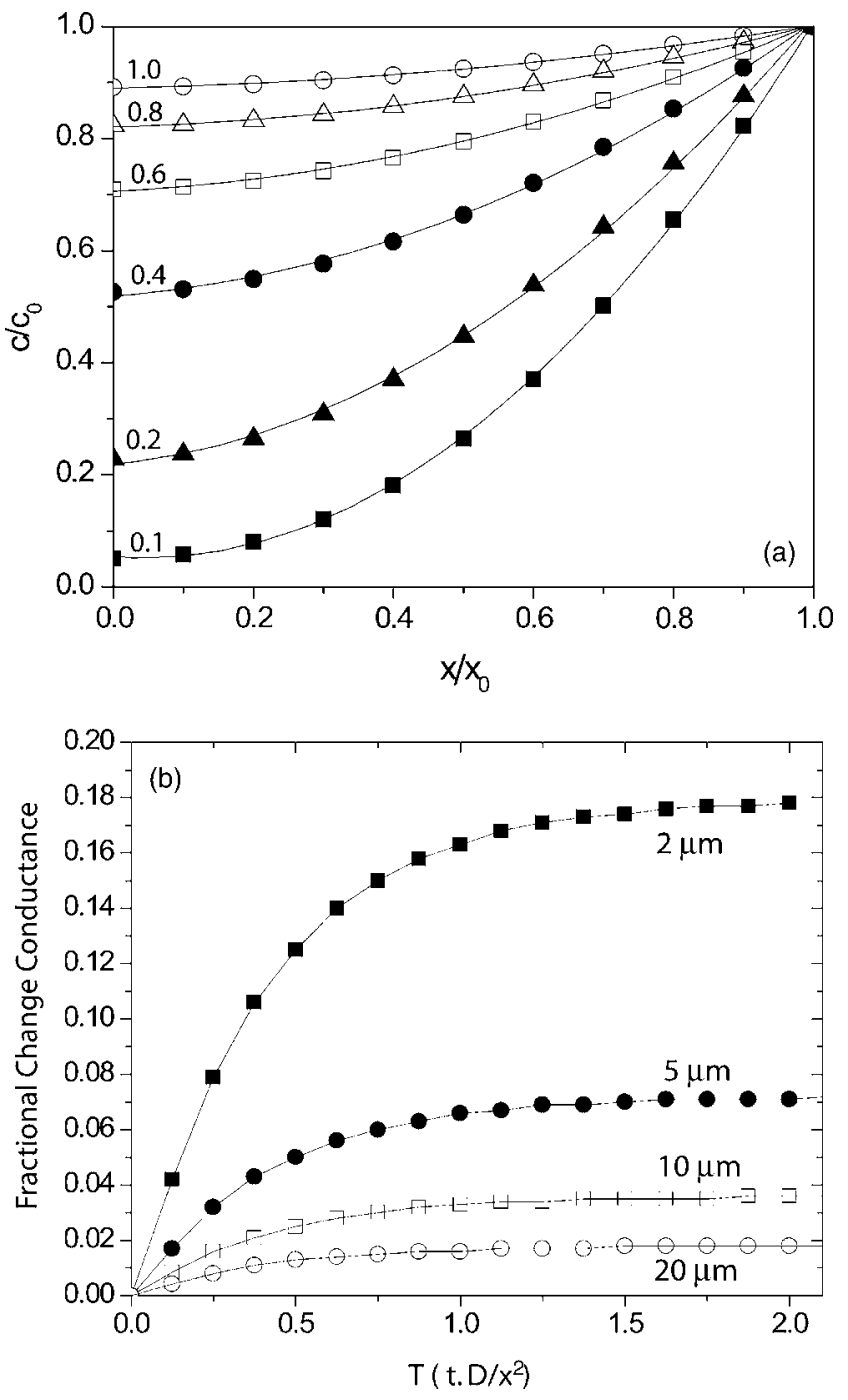

FIG. 2. (a) Gas concentration profile at various times inside the nano-tinoxide films coated on the MEMS device; (b) analytical solution to Eq. (9) showing the effect of electrode spacing on change in conductance inside the film.

$$
i(t)=\int J d s=\int \sigma E d s .
$$

The change in total current $[i(t)]$ flowing through the device can be given by

$$
\Delta i(t)=\mu e k \int_{c s} c E d s
$$

where $c$ is the gas concentration, $E$ is the electric field inside the film, while $\mu$ and $e$ are the electron mobility and charge, respectively.

The change in electric conductance is given by ${ }^{10}$

$$
\Delta G(t)=\Delta i(t) / V,
$$

where $V$ is the applied voltage.

The solution for Laplace's equation for the semiconducting layer above the coplanar electrode is derived in detail elsewhere as ${ }^{10}$

$$
\Delta G / G_{0}=\int_{0}^{1} C\left[(1-X)^{2}+g^{2} / 4 x_{0}^{2}\right]^{-1 / 2} d x,
$$

where $G_{0}$ is the initial conductance of the film. 

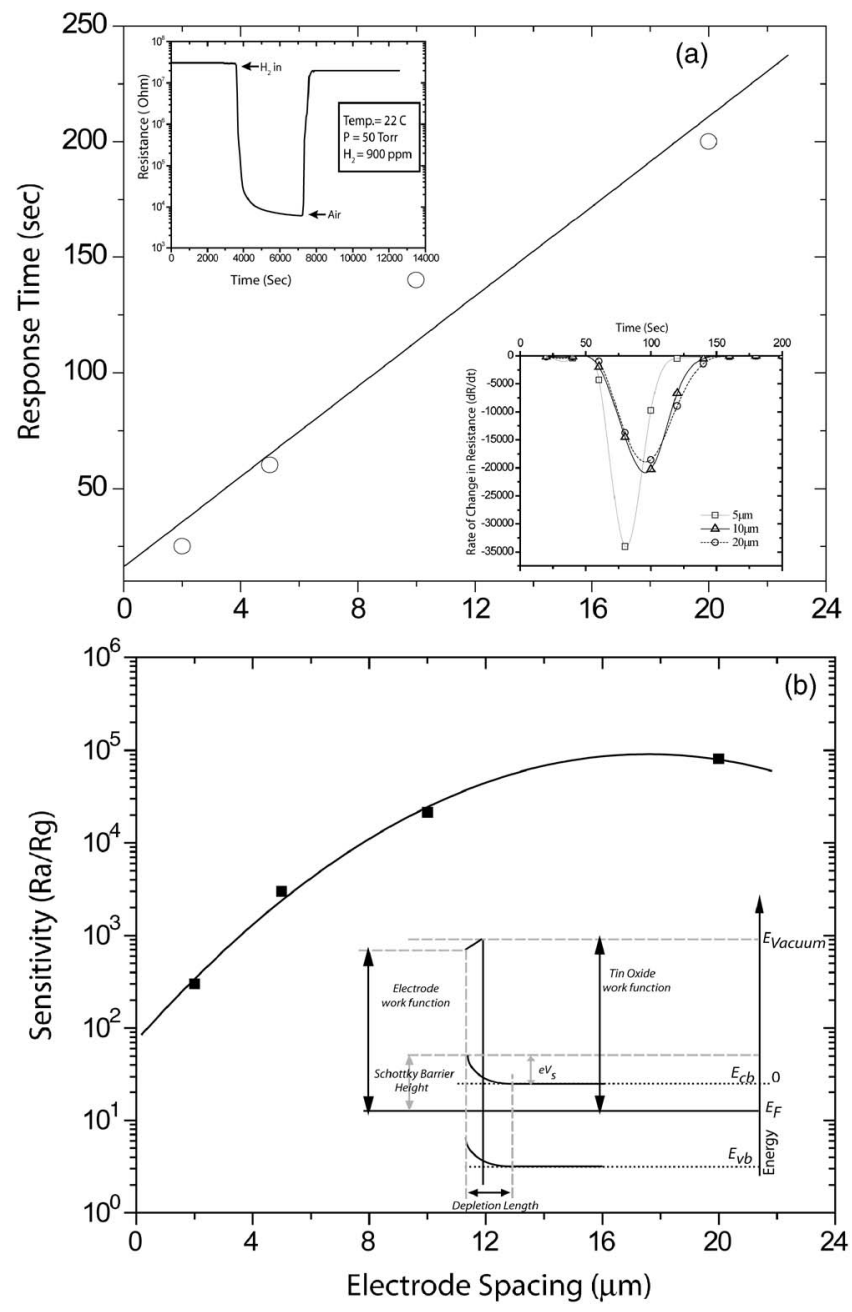

FIG. 3. (a) Figure showing the effect of electrode spacing variation on the response time of the nano-tin-oxide coated MEMS devices towards $900 \mathrm{ppm}$ of hydrogen at room conditions; (a-i) inset showing typical response transient nano-tin-oxide coated MEMS sensor; (a-ii) inset showing the effect of electrode spacing on the rate of change of resistance inside the nano-tin-oxide coated thin film hydrogen sensor. (b) Impact of electrode spacing on gas sensitivity of the room temperature nano-tin-oxide hydrogen sensor.

The theoretical transient response for the current electrode geometry where the fractional change in conductance is plotted as function of factor $T$ is shown in Fig. 2(b) for different electrode spacings. From the figure it can be interpreted that the response of the device depends on the ratio $g / x_{0}$. At a constant thickness $\left(x_{0}\right)$, the sensor response is solely controlled by the electrode spacings. Smaller gap size leads to faster change in the electric field inside the film resulting in faster response of the device.

Figure 3(a) shows the effect of electrode spacing $(g)$ on the response time of the MEMS hydrogen sensors. From the figure it can be concluded that the sensor response time increases linearly with the electrode spacing. The inset in Fig. 3(a-i) shows a response transient of a sensor (electrode spacing $=5 \mu \mathrm{m}$ and number of fingers $=8$ ) when exposed to hydrogen $(900 \mathrm{ppm})$ at room temperature. The sensor with
$5 \mu \mathrm{m}$ electrode spacing showed a faster rate of change of resistance (higher conductance change) as compared to 10 and $20 \mu \mathrm{m}$ electrode spaced MEMS sensors as shown in Fig. 3(a-ii). These experimental results are in good agreement with the theoretically modeled behavior of the effective variation in the electrode spacing on the sensor response. Change in spacing between the electrodes also changes the sensitivity of the sensor. Figure 3(b) shows the sensitivity of a nanodoped tin oxide MEMS based hydrogen sensor. Increase in spacing from 2 to $20 \mu \mathrm{m}$ results in an increase in active sensing area by almost $10 \%$ for a pair of electrodes. This results in the increase of sensitivity $(S=R a / R g)$ from 500 (for $2 \mu \mathrm{m}$ spacing) to 80000 (for $20 \mu \mathrm{m}$ spacing) for $900 \mathrm{ppm} \mathrm{H}_{2}$. When the sensor is exposed to hydrogen, gas molecules are adsorbed not only on the surface of the $\mathrm{SnO}_{2}$ but also on the interface of the tin oxide-electrode $(\mathrm{Au})$ interface. This results in an increase in the thickness of the electron-depleted layer of $\mathrm{SnO}_{2}$ grain as well as in the work function of Au. As a result a large increase in the Schottky barrier height at the $\mathrm{Au}-\mathrm{SnO}_{2}$ interface is expected [inset of Fig. 3(b)]. Due to a very high increase in the active sensing area, the electrode gap increases the number of charge carriers (free electrons) after reacting with hydrogen, which results in the compensation of Schottky barrier height. Therefore, an increase in electrode spacing shows higher gas sensitivity.

This letter successfully demonstrated the application of diffusion reaction model for enlightening the impact of electrode spacing on response kinetics of the nanomicrointegrated hydrogen sensor at room temperature. The faster response shown by the smaller electrode spacing is due to the reduced path length for free electrons on the nanoparticle surface when exposed to hydrogen. Our current results indicate that by optimizing the device architecture, it is possible to tune the sensitivity and response time, thus the overall sensing kinetics at room temperature.

The authors thank NASA ASRC, NASA Glenn (NAG: 32751), and NSF EEC 0453436 for funding the research. One of the authors (N.P.) is a REU student (2006).

${ }^{1}$ I. Simon, N. Barasan, M. Bauer, and U. Weimar, Sens. Actuators B 73, 1 (2001).

${ }^{2}$ N. Barsan and U. Weimar, J. Electroceram. 7, 143 (2001).

${ }^{3}$ S. Capone, P. Siciliano, F. Quaranta, R. Rella, M. Epifani, and L. Vasanelli, Sens. Actuators B 77, 503 (2001).

${ }^{4} \mathrm{~S}$. Shukla and S. Seal, in Encyclopedia of Nanoscience and Nanotechnology, edited by H. S. Nalwa (American Science, Valencia, CA, 2005), 10, pp. 27-42.

${ }^{5}$ M.-I. Baraton, L. Merhari, H. Ferkel, and J.-F. Castagnet, Mater. Sci. Eng., C 19, 315 (2002).

${ }^{6}$ C. Drake, S. Deshpande, and S. Seal, Appl. Phys. Lett. 89, 143116 (2006).

${ }^{7}$ S. Shukla, S. Seal, L. Ludwig, and C. Perrish, Sens. Actuators B 97, 256 (2004).

${ }^{8}$ J. Crank, The Mathematics of Diffusion, 1st ed. (Clarendon, Oxford, UK, 1955), Chap. 4, pp. 42-62.

${ }^{9} \mathrm{C}$. Liu, Foundation of MEMS, 1st ed. (Pearson Education, Upper Saddle River, NJ, 2006), Chap. 3, pp. 48-103.

${ }^{10}$ J. W. Gardner, Semicond. Sci. Technol. 4, 345 (1989). 